\title{
Floquet engineering of the Luttinger Hamiltonian
}

\author{
O. V. Kibis ${ }^{1}$ - M. V. Boev ${ }^{1}$, V. M. Kovalev ${ }^{1}$, and I. A. Shelykh ${ }^{2,3}$ \\ ${ }^{1}$ Department of Applied and Theoretical Physics, Novosibirsk State Technical University, \\ Karl Marx Avenue 20, Novosibirsk 630073, Russia \\ ${ }^{2}$ Science Institute, University of Iceland, Dunhagi 3, IS-107, Reykjavik, Iceland and \\ ${ }^{3}$ Department of Physics and Engineering, ITMO University, Saint Petersburg 197101, Russia
}

\begin{abstract}
Within the Floquet theory of periodically driven quantum systems, we developed the theory of light-induced modification of electronic states in semiconductor materials described by the Luttinger Hamiltonian (the electronic term $\Gamma_{8}$ ). Particularly, exact solutions of the Floquet problem are found for the band edge in the cases of linearly and circularly polarized irradiation. It is shown that the irradiation changes electron effective masses near the band edge, induces anisotropy of the electron dispersion and splits the bands. It is demonstrated that the light-induced band splitting strongly depends on the light polarization. Namely, the circularly polarized light acts similarly to a stationary magnetic field and lifts the spin degeneracy of electron branches, whereas a linearly polarized light does not affect the spin degeneracy and only splits the bands in the center of the Brillouin zone. The present theory can be applied to describe electronic properties of various semiconductor structures irradiated by an electromagnetic field in the broad frequency range.
\end{abstract}

\section{INTRODUCTION}

During last years, the control of electronic parameters of condensed-matter structures by an electromagnetic field (so-called "Floquet engineering" based on the Floquet theory for periodically driven quantum systems) became the important and established research area which resulted in the discovery of many fundamental effects (see, e.g., Refs. 1 6). It is well known that absorption of an electromagnetic field by an electron system takes place if only a characteristic electronic frequency coincides with a field frequency (resonant field). However, even if the field is non-resonant and cannot be absorbed, it still interacts with electrons. Formally, this non-resonant interaction is described by the nonstationary Schrödinger equation with a periodically time-dependent potential. Solution of this equation is the Floquet wave function which is periodic in time with period of the field. Averaging all electronic characteristics obtained with using the Floquet function over the field period, one can construct the quantum dynamics equations for electrons "dressed" by the field, which are similar to the equations for "bare" electrons but depend on field parameters. As a consequence, behavior of dressed electrons can be considered by analogy with the behavior of bare electrons, stationary physical parameters of which (energy spectrum, effective mass, etc.) are renormalized by the field. Therefore, the theory of renormalization of electronic properties of any structure by an electromagnetic field (Floquet engineering) is based on solution of the Floquet problem for the corresponding nonstationary Schrödinger equation.

Historically, investigations of the processes of strong interaction of electrons with an electromagnetic field, which lead to stationary renormalization of physical properties of electronic systems by the field, have started in the middle of 20th century. For a long time, the main objects of the investigations were atomic and molecular systems. In particular, the first investigations of non-resonant interaction of electrons with a strong field were carried out for isolated atoms and lead to observation of the atomic energy levels shift caused by light (the Autler-Townes effect) $)^{7}, \underline{8}$. As to investigations of these effects in solid state structures, they have started with the works done by Galitsky, Goreslavsky and Elesin ${ }^{9}, 10$, who theoretically predicted the existence of light-induced band gaps in energy spectrum of semiconductors, which later were observed experimentally $\stackrel{11}{ }$. Their pioneering ideas about light-induced modification of band electronic structure of solids were developed later theoretically and experimentally for various crystal structures $12-18$. However, effects of electromagnetic renormalization of electronic properties of solids were ignored as rule in the most studies for a long time because the scattering of conduction electrons significantly obstructs experimental investigations of them. Situation has changed when it became possible to fabricate solid state structures with very high charge carrier mobility and, correspondingly, with weak electron scattering. As a consequence, during last decade were published many works dedicated to the Floquet engineering of various solid state structures, including quantum rings $\frac{19}{2}-25$, quantum wells $\underline{26}-\underline{22}$, topological insulators ${ }^{33}-39$, graphene and related $2 \mathrm{D}$ materials $\underline{40}-\underline{49}$, etc.

Among various condensed-matter structures important to both fundamental science and device applications, it should be noted especially those of them which are based on conventional semiconductor materials ( $\mathrm{Si}, \mathrm{Ge}$ and $\mathrm{A}_{3} \mathrm{~B}_{5}$ semiconductors) and gapless semiconductors (HgTe and related materials). Particularly, the most of modern nanostructures are fabricated with using them. Since valence band of the conventional semiconductors and band structure of the gapless semiconductors near the band edge (the electronic term $\Gamma_{8}$ in the $\Gamma$ point of the Brillouin zone) are described by the well-known Luttinger Hamiltonian ${ }^{50}$, it is necessary to develop the consistent Floquet theory for electronic systems described by the Hamiltonian in order to control electronic properties of the corresponding semiconductor structures by an electromagnetic field. The present work is dedicated 
to solving this theoretical problem.

The article is organized as follows. In Section II, we solved the Floquet problem for the nonstationary Schrödinger equation based on the Luttinger Hamiltonian in the presence of an electromagnetic field. In Section III, we calculated electron dispersion of the Luttinger Hamiltonian modified by the field and discussed possible experimental manifestations of the field-induced renormalization of electronic properties. The last sections of the article contain conclusion and acknowledgments.

\section{MODEL}

Let us consider a semiconductor material with the electron energy spectrum described by the Luttinger Hamiltonian (the electronic term $\Gamma_{8}$ ), which is irradiated by a plane electromagnetic wave with the frequency $\omega$ and the electric field amplitude $E_{0}$ (see Fig. 1). Assuming size of the irradiated semiconductor sample along the direction of the wave propagation, $d$, to be much larger than the interatomic distance and much less than the wave length, $\lambda=2 \pi c / \omega$, we can neglect the size quantization of electron energy spectrum of the sample and consider the wave field inside the sample as uniform. Then electronic states of the irradiated semiconductor sample near the center of the Brillouin zone (the electronic term $\Gamma_{8}$ ) within the conventional minimal coupling approach can be described by the time-dependent Hamiltonian,

$$
\hat{\mathcal{H}}(\mathbf{k}, t)=\hat{\mathcal{H}}_{L}(\mathbf{k}-e \mathbf{A}(t) / \hbar),
$$

where $\hat{\mathcal{H}}_{L}(\mathbf{k})$ is the Luttinger Hamiltonian, $\mathbf{k}=$ $\left(k_{x}, k_{y}, k_{z}\right)$ is the electron wave vector, and $\mathbf{A}(t)=$ $\left(A_{x}, A_{y}, A_{z}\right)$ is the vector potential of the wave inside the semiconductor, which periodically depends on the time, $t$. In the present analysis, we will restrict the consideration to the isotropic approximation of the electron dispersion in the semiconductor. Then the Luttinger Hamiltonian takes the form 51,52

$$
\hat{\mathcal{H}}_{\mathrm{L}}(\mathbf{k})=\left(\gamma_{1}+5 \gamma / 2\right) \mathbf{k}^{2}-2 \gamma(\mathbf{k J})^{2}
$$

where $\gamma=\left(2 \gamma_{2}+3 \gamma_{3}\right) / 5, \gamma_{1,2,3}$ are the Luttinger parameters, and $J_{x, y, z}$ are the $4 \times 4$ matrices corresponding to the electron angular momentum $J=3 / 2$. To perform calculations, it is convenient to rewrite the Hamiltonian (2) as a $4 \times 4$ matrix in the basis of Luttinger-Kohn wave functions, $\psi_{j_{z}}$, which describe four-fold degenerate electron states of the conduction and valence band in the center of the bulk Brillouin zone (the $\Gamma$ point), and correspond to the four different projections of electron momentum on the $z$ axis, $j_{z}= \pm 1 / 2$ and $j_{z}= \pm 3 / 2$ (see for more details, e.g., the appendix in Ref. 53). In this basis, the
Hamiltonian (2) reads

$$
\hat{\mathcal{H}}(\mathbf{k})=\begin{array}{|c||cccc|}
\hline j_{z} \backslash j_{z} & +3 / 2 & +1 / 2 & -1 / 2 & -3 / 2 \\
\hline+3 / 2 & F & H & I & 0 \\
+1 / 2 & H^{*} & G & 0 & I \\
-1 / 2 & I^{*} & 0 & G & -H \\
-3 / 2 & 0 & I^{*} & -H^{*} & F \\
\hline
\end{array}
$$

where the matrix elements are

$$
\begin{aligned}
F & =\left(\gamma_{1}+\gamma\right)\left(k_{x}^{2}+k_{y}^{2}\right)+\left(\gamma_{1}-2 \gamma\right) k_{z}^{2}, \\
G & =\left(\gamma_{1}-\gamma\right)\left(k_{x}^{2}+k_{y}^{2}\right)+\left(\gamma_{1}+2 \gamma\right) k_{z}^{2}, \\
I & =-\sqrt{3} \gamma\left(k_{x}-i k_{y}\right)^{2}, \\
H & =-2 \sqrt{3} \gamma\left(k_{x}-i k_{y}\right) k_{z} .
\end{aligned}
$$

In the following, we will demonstrate that electronic properties of an irradiated semiconductor substantially depend on polarization of the electromagnetic wave. Therefore, it is convenient to analyze the Hamiltonian (3) for the linear and circular polarizations separately.

Linear polarization. Let the electromagnetic wave propagates along the $x$ axis and is linearly polarized along the $z$ axis (see Fig. 1a). Then its vector potential inside the semiconductor can be written as
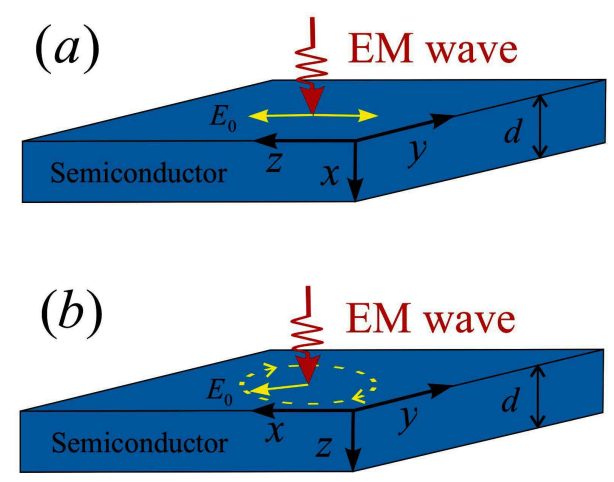

FIG. 1: Sketch of the semiconductor sample irradiated by an electromagnetic wave with different polarizations: (a) linear polarization; (b) circular polarization.

$$
\mathbf{A}(t)=\left(0,0, \frac{E_{0}}{\omega} \cos \omega t\right)
$$

To simplify calculations, let us subject the Hamiltonian (1) with the vector potential (5) to the unitary transfor- 
mation

$$
\hat{U}=\frac{1}{\sqrt{2}}\left[\begin{array}{cccc}
e^{i \phi} & 0 & 0 & e^{i \phi} \\
0 & e^{i \varphi} & e^{i \varphi} & 0 \\
0 & -e^{-i \varphi} & e^{-i \varphi} & 0 \\
-e^{-i \phi} & 0 & 0 & e^{-i \phi}
\end{array}\right]
$$

where $\phi=-3 \theta / 2+\pi / 4, \varphi=-\theta / 2-\pi / 4, \theta$ is the polar angle of the electron wave vector in the $(x, y)$ plane and the electron wave vector as a function of the angle $\theta$ reads

$$
\mathbf{k}=\left(k_{x}, k_{y}, k_{z}\right)=\left(\sqrt{k_{x}^{2}+k_{y}^{2}} \cos \theta, \sqrt{k_{x}^{2}+k_{y}^{2}} \sin \theta, k_{z}\right) .
$$

Then the transformed Hamiltonian (1), $\hat{\mathcal{H}}^{\prime}(\mathbf{k}, t)=$ $\hat{U}^{\dagger} \hat{\mathcal{H}}(\mathbf{k}, t) \hat{U}$, takes the block-diagonal form,

$$
\hat{\mathcal{H}}^{\prime}(\mathbf{k}, t)=\left[\begin{array}{cc}
\hat{\mathcal{H}}^{(1)}(\mathbf{k}, t) & 0 \\
0 & \hat{\mathcal{H}}^{(2)}(\mathbf{k}, t)
\end{array}\right],
$$

where the $2 \times 2$ matrices are

$$
\hat{\mathcal{H}}^{(1)}(\mathbf{k}, t)=\left[\begin{array}{cc}
\widetilde{F} & \widetilde{M} \\
\widetilde{M}^{*} & \widetilde{G}
\end{array}\right], \hat{\mathcal{H}}^{(2)}(\mathbf{k}, t)=\left[\begin{array}{cc}
\widetilde{G} & -\widetilde{M} \\
-\widetilde{M}^{*} & \widetilde{F}
\end{array}\right]
$$

and the matrix elements of the Hamiltonian are

$$
\begin{aligned}
\widetilde{F} & =F+\left(\gamma_{1}-2 \gamma\right)\left(\frac{e E_{0}}{\hbar \omega} \cos \omega t-2 k_{z}\right) \frac{e E_{0}}{\hbar \omega} \cos \omega t \\
\widetilde{G} & =G+\left(\gamma_{1}+2 \gamma\right)\left(\frac{e E_{0}}{\hbar \omega} \cos \omega t-2 k_{z}\right) \frac{e E_{0}}{\hbar \omega} \cos \omega t, \\
\widetilde{M} & =\frac{\gamma}{|\gamma|}|I|+i \frac{\gamma}{|\gamma|} \frac{|H|}{\left|k_{z}\right|}\left[k_{z}-\left(\frac{e E_{0}}{\hbar \omega}\right) \cos \omega t\right] .
\end{aligned}
$$

In the most general form, the nonstationary Schrödinger equation for an electron in a periodically time-dependent field with the frequency $\omega$ can be written as $i \hbar \partial_{t} \psi(t)=\hat{\mathcal{H}}(t) \psi(t)$, where $\hat{\mathcal{H}}(t+T)=\hat{\mathcal{H}}(t)$ is the periodically time-dependent Hamiltonian and $T=2 \pi / \omega$ is the field period. It follows from the Floquet theorem that solution of the Schrödinger equation is the Floquet function, $\psi(t)=e^{-i \varepsilon t / \hbar} \varphi(t)$, where $\varphi(t+T)=\varphi(t)$ is the periodically time-dependent function and $\varepsilon$ is the electron (quasi)energy describing behavior of the electron in the periodical field ${ }^{1-6}$. The Floquet problem is aimed to find the electron energy spectrum, $\varepsilon$. Let us solve the problem for the Hamiltonian (7).

The two Hamiltonians (8) describe the two spindegenerated electron states of the semiconductor with the same energy, $\varepsilon(\mathbf{k})$. Therefore, one can consider any of the two Hamiltonians $\hat{\mathcal{H}}^{(1,2)}(\mathbf{k}, t)$ to find the sought energy spectrum of the irradiated semiconductor, $\varepsilon(\mathbf{k})$. For definiteness, let us restrict the consideration by the $2 \times 2$ matrix Hamiltonian $\hat{\mathcal{H}}^{(1)}(\mathbf{k}, t)$. The Floquet problem with this Hamiltonian can be solved accurately at $\mathbf{k}=0$. Namely, the Hamiltonian $\hat{\mathcal{H}}^{(1)}(\mathbf{k}, t)$ at $\mathbf{k}=0$ reads

$$
\hat{\mathcal{H}}_{0}^{(1)}(t)=\left(\frac{e E_{0}}{\hbar \omega}\right)^{2} \cos ^{2} \omega t\left[\begin{array}{cc}
\gamma_{1}-2 \gamma & 0 \\
0 & \gamma_{1}+2 \gamma
\end{array}\right] .
$$

Exact solutions of the Schrödinger equation with the Hamiltonian (9), $i \hbar \partial_{t} \Phi_{ \pm}=\hat{\mathcal{H}}_{0}^{(1)}(t) \Phi_{ \pm}$, are the two Floquet functions $\Phi_{ \pm}=\exp \left(-i \varepsilon_{ \pm} t / \hbar\right) \phi_{ \pm}$, where

$$
\begin{aligned}
& \phi_{-}=\left[\begin{array}{c}
e^{-i\left[\left(\gamma_{1}-2 \gamma\right) / 4 \hbar \omega\right]\left(e E_{0} / \hbar \omega\right)^{2} \sin 2 \omega t} \\
0
\end{array}\right], \\
& \phi_{+}=\left[\begin{array}{c}
0 \\
e^{-i\left[\left(\gamma_{1}+2 \gamma\right) / 4 \hbar \omega\right]\left(e E_{0} / \hbar \omega\right)^{2} \sin 2 \omega t}
\end{array}\right],
\end{aligned}
$$

are the eigenspinors of the Floquet problem with the Hamiltonian (9) and the corresponding electron energies at $\mathbf{k}=0$ are

$$
\varepsilon_{ \pm}=\frac{\gamma_{1} \pm 2 \gamma}{2}\left(\frac{e E_{0}}{\hbar \omega}\right)^{2}
$$

Eqs. (10)-111) describe the exact solutions of the Floquet problem with the Luttinger Hamiltonian at the band edge $(\mathbf{k}=0)$ in the case of linearly polarized irradiation. As to the Floquet problem for $\mathbf{k} \neq 0$, it can be solved approximately for small electron wave vectors, $\mathbf{k}$, as follows. In the new orthonormal basis (10), the Hamiltonian $\hat{\mathcal{H}}^{(1)}(\mathbf{k}, t)$ reads

$$
\hat{\mathcal{H}}^{(1)}(\mathbf{k}, t)=\left[\begin{array}{cc}
\bar{F} & \bar{M} \\
\bar{M} & \bar{G}
\end{array}\right],
$$

where its matrix elements are

$$
\begin{aligned}
\bar{F} & =\varepsilon_{-}+F-2\left(\gamma_{1}-2 \gamma\right) k_{z}\left(\frac{e E_{0}}{\hbar \omega}\right) \cos \omega t, \\
\bar{G} & =\varepsilon_{+}+G-2\left(\gamma_{1}+2 \gamma\right) k_{z}\left(\frac{e E_{0}}{\hbar \omega}\right) \cos \omega t, \\
\bar{M} & =\widetilde{M} e^{-i \eta \sin 2 \omega t}
\end{aligned}
$$

and $\eta=(\gamma / \hbar \omega)\left(e E_{0} / \hbar \omega\right)^{2}$. To find the energy spectrum, $\varepsilon(\mathbf{k})$, for small electron wave vectors $\mathbf{k}$, one can apply the conventional perturbation theory for periodically driven quantum systems ${ }^{1-5}$ to the time-dependent Hamiltonian (12). As a result, we arrive at the effective time-independent Hamiltonian,

$$
\hat{\mathcal{H}}_{\text {eff }}(\mathbf{k})=\frac{1}{T} \int_{0}^{T} \hat{\mathcal{H}}^{(1)}(\mathbf{k}, t) d t
$$

which is correct for small electron wave vectors, $\mathbf{k}$, satisfying the condition $\mathbf{k}^{2} \ll \hbar \omega /|\gamma|$. Using the well-known Jakobi-Anger expansion, $e^{i z \sin \gamma}=\sum_{n=-\infty}^{\infty} J_{n}(z) e^{i n \gamma}$, to transform the exponential factor in the Hamiltonian (12), the effective Hamiltonian (13) can be rewritten in the explicit form as

$$
\begin{aligned}
& \hat{\mathcal{H}}_{\mathrm{eff}}(\mathbf{k})= \\
& {\left[\begin{array}{cc}
\varepsilon_{-}+F & \frac{\gamma}{|\gamma|}\left[|I|+i \frac{k_{z}}{\left|k_{z}\right|}|H|\right] J_{0}(\eta) \\
\frac{\gamma}{|\gamma|}\left[|I|-i \frac{k_{z}}{\left|k_{z}\right|}|H|\right] J_{0}(\eta) & \varepsilon_{+}+G
\end{array}\right],}
\end{aligned}
$$


where $J_{0}(\eta)$ is the zeroth order Bessel function of the first kind. Diagonalizing the effective Hamiltonian (14), we arrive at the sought electron energy spectrum near the $\Gamma$ point of the Brillouin zone,

$$
\begin{aligned}
& \varepsilon^{( \pm)}(\mathbf{k})=\left(\gamma_{1} / 2\right)\left(e E_{0} / \hbar \omega\right)^{2}+\gamma_{1} k^{2} \pm \gamma\left[\left(2 k_{z}^{2}-k_{x}^{2}-k_{y}^{2}\right.\right. \\
& \left.\left.+\left[e E_{0} / \hbar \omega\right]^{2}\right)^{2}+3\left(k_{x}^{2}+k_{y}^{2}\right)\left(k_{x}^{2}+k_{y}^{2}+4 k_{z}^{2}\right) J_{0}^{2}(\eta)\right]^{1 / 2}
\end{aligned}
$$

where the signs " \pm " correspond to the two branches of the Luttinger Hamiltonian. It should be stressed that Eq. (15) correctly describes the electron energy spectrum near the band edge $\left(\mathbf{k}^{2} \ll \hbar \omega /|\gamma|\right)$ for any field frequency $\omega$. Certainly, the energy spectrum (15) in the absence of the irradiation $\left(E_{0}=0\right)$ exactly coincides with the spectrum of the unperturbed Luttinger Hamiltonian (2), $\varepsilon^{( \pm)}(\mathbf{k})=\left(\gamma_{1} \pm 2 \gamma\right) k^{2}$.

Circular polarization. Let an electromagnetic wave irradiating a semiconductor propagates along the $z$ axis and is circularly polarized in the $(x, y)$ plane (see Fig. 1b). Then its vector potential inside the semiconductor can be written as

$$
\mathbf{A}=\left(\frac{E_{0}}{\omega} \cos \omega t, \frac{E_{0}}{\omega} \sin \omega t, 0\right) .
$$

The Floquet problem with the Luttinger Hamiltonian (1) and the vector potential (16) can be solved accurately in the particular case of $\mathbf{k}=0$ as follows. Taking into account the Luttinger Hamiltonian matrix (3), the considered Hamiltonian (1) with the vector potential (16) at $\mathbf{k}=0$ can be written in the block-diagonal form as

$$
\hat{\mathcal{H}}_{0}=\left[\begin{array}{cc}
\hat{\mathcal{H}}^{(+)} & 0 \\
0 & \hat{\mathcal{H}}^{(-)}
\end{array}\right]
$$

where the Hamiltonian $\hat{\mathcal{H}}_{0}^{( \pm)}$written in the basis $\left\{\psi_{ \pm 3 / 2}, \psi_{\mp 1 / 2}\right\}$ reads

$$
\hat{\mathcal{H}}^{( \pm)}=\left(\frac{e E_{0}}{\hbar \omega}\right)^{2}\left[\begin{array}{cc}
\gamma_{1}+\gamma & -\sqrt{3} \gamma e^{\mp i 2 \omega t} \\
-\sqrt{3} \gamma e^{ \pm i 2 \omega t} & \gamma_{1}-\gamma
\end{array}\right] .
$$

Solving the nonstationary Schrödinger equation with the Hamiltonian (18), $i \hbar \partial_{t} \Phi_{1,2}^{( \pm)}=\hat{\mathcal{H}}^{( \pm)} \Phi_{1,2}^{( \pm)}$, one can find the four exact Floquet functions, $\Phi_{1,2}^{( \pm)}=$ $\exp \left(-i \varepsilon_{1,2}^{( \pm)} t / \hbar\right) \phi_{1,2}^{( \pm)}$, and the four eigenspinors of the considered Floquet problem,

$$
\begin{aligned}
& \phi_{1}^{( \pm)}=\left[\begin{array}{c}
\frac{\gamma}{|\gamma|} \sqrt{\frac{\Omega_{ \pm}-\Delta_{ \pm}}{2 \Omega_{ \pm}}} e^{\mp i \omega t} \\
\sqrt{\frac{\Omega_{ \pm}+\Delta_{ \pm}}{2 \Omega_{ \pm}}} e^{ \pm i \omega t}
\end{array}\right] e^{i \omega t}, \\
& \phi_{2}^{( \pm)}=\left[\begin{array}{c}
\frac{\gamma}{|\gamma|} \sqrt{\frac{\Omega_{ \pm}+\Delta_{ \pm}}{2 \Omega_{ \pm}}} e^{\mp i \omega t} \\
-\sqrt{\frac{\Omega_{ \pm}-\Delta_{ \pm}}{2 \Omega_{ \pm}}} e^{ \pm i \omega t}
\end{array}\right] e^{-i \omega t},
\end{aligned}
$$

where $\Omega_{ \pm}=\sqrt{\Delta_{ \pm}^{2}+3 \gamma^{2}\left(e E_{0} / \hbar \omega\right)^{4}}, \quad \Delta_{ \pm}=$ $\gamma\left(e E_{0} / \hbar \omega\right)^{2} \mp \hbar \omega$, and the corresponding electron energies at $\mathbf{k}=0$ are

$$
\begin{aligned}
& \varepsilon_{1}^{( \pm)}=\gamma_{1}\left(e E_{0} / \hbar \omega\right)^{2}+\hbar \omega-\Omega_{ \pm}, \\
& \varepsilon_{2}^{( \pm)}=\gamma_{1}\left(e E_{0} / \hbar \omega\right)^{2}-\hbar \omega+\Omega_{ \pm} .
\end{aligned}
$$

Eqs. (19)-(20) describe the exact solutions of the Floquet problem with the Luttinger Hamiltonian at the band edge $(\mathbf{k}=0)$ in the case of circularly polarized irradiation. To solve the Floquet problem with the Luttinger Hamiltonian (11) and the vector potential (16) at $\mathbf{k} \neq 0$, let us rewrite the Hamiltonian in the new orthonormal basis (19). For small electron wave vectors, k, satisfying the condition $\mathbf{k}^{2} \ll \hbar \omega /|\gamma|$, one can apply the conventional perturbation theory to the rewritten Hamiltonian in the way discussed above for a linearly polarized field. As a result, we arrive at the effective time-independent Hamiltonian, $\hat{\mathcal{H}}_{\text {eff }}(\mathbf{k})$, which is similar to the Hamiltonian (13). Namely, the Hamiltonian $\hat{\mathcal{H}}_{\mathrm{eff}}(\mathbf{k})$ is the Hamiltonian (1) with the vector potential (16), which is rewritten in the basis (19) and time-averaged over the field period. In the explicit form, the effective Hamiltonian describing the sought electron energy spectrum, $\varepsilon(\mathbf{k})$, at small wave vectors $\left(\mathbf{k}^{2} \ll \hbar \omega /|\gamma|\right)$ reads

$$
\hat{\mathcal{H}}_{\text {eff }}(\mathbf{k})=\begin{array}{|c||cccc|}
\hline \phi_{j}^{( \pm)} \backslash \phi_{j}^{( \pm)} & \phi_{1}^{(+)} & \phi_{1}^{(-)} & \phi_{2}^{(+)} & \phi_{2}^{(-)} \\
\hline \hline \phi_{1}^{(+)} & A_{+} & -C_{+} & D_{+} & 0 \\
\phi_{1}^{(-)} & -C_{+}^{*} & A_{-} & 0 & -D_{-} \\
\phi_{2}^{(+)} & D_{+}^{*} & 0 & B_{+} & C_{-} \\
\phi_{2}^{(-)} & 0 & -D_{-}^{*} & C_{-}^{*} & B_{-} \\
\hline
\end{array}
$$

where the matrix elements are

$$
\begin{aligned}
A_{ \pm} & =\frac{\Omega_{ \pm}-\Delta_{ \pm}}{2 \Omega_{ \pm}} F+\frac{\Omega_{ \pm}+\Delta_{ \pm}}{2 \Omega_{ \pm}} G+\varepsilon_{1}^{( \pm)}, \\
B_{ \pm} & =\frac{\Omega_{ \pm}+\Delta_{ \pm}}{2 \Omega_{ \pm}} F+\frac{\Omega_{ \pm}-\Delta_{ \pm}}{2 \Omega_{ \pm}} G+\varepsilon_{2}^{( \pm)}, \\
C_{ \pm} & =\frac{\gamma}{|\gamma|}\left[\sqrt{\frac{\Omega_{+} \mp \Delta_{+}}{2 \Omega_{+}}} \sqrt{\frac{\Omega_{-} \pm \Delta_{-}}{2 \Omega_{-}}}\right. \\
& \left.-\sqrt{\frac{\Omega_{-} \mp \Delta_{-}}{2 \Omega_{-}}} \sqrt{\frac{\Omega_{+} \pm \Delta_{+}}{2 \Omega_{+}}}\right] H, \\
D_{ \pm} & =\frac{\gamma}{|\gamma|} \frac{\Omega_{ \pm} \mp \Delta_{ \pm}}{2 \Omega_{ \pm}} I .
\end{aligned}
$$

Correspondingly, the energy spectrum, $\varepsilon(\mathbf{k})$, can be found as a solution of the secular equation,

$$
\operatorname{det}\left[\hat{\mathcal{H}}_{\mathrm{eff}}(\mathbf{k})-\mathbf{1} \varepsilon(\mathbf{k})\right]=0,
$$

where $\mathbf{1}$ is the unity matrix. To find the spectrum in the broad range of electron wave vectors, the equation (23) should be solved numerically. However, the spectrum $\varepsilon(\mathbf{k})$ can be written in analytical form for the highsymmetry directions in the Brillouin zone, $\mathbf{k}=\left(0,0, k_{z}\right)$ and $\mathbf{k}=\left(k_{x}, k_{y}, 0\right)$. Namely, we can write the spectrum as the four branches, $\varepsilon_{1,2}^{( \pm)}\left(k_{z}\right)$ for $k_{x}=k_{y}=0$ 
and $\varepsilon_{1,2}^{( \pm)}\left(k_{x}, k_{y}\right)$ for $k_{z}=0$, where

$$
\begin{aligned}
& \varepsilon_{1}^{( \pm)}\left(k_{z}\right)=\gamma_{1}\left(\frac{e E_{0}}{\hbar \omega}\right)^{2}+\hbar \omega-\Omega_{ \pm}+\gamma_{1} k_{z}^{2}+\frac{2 \gamma k_{z}^{2} \Delta_{ \pm}}{\Omega_{ \pm}}, \\
& \varepsilon_{2}^{( \pm)}\left(k_{z}\right)=\gamma_{1}\left(\frac{e E_{0}}{\hbar \omega}\right)^{2}-\hbar \omega+\Omega_{ \pm}+\gamma_{1} k_{z}^{2}-\frac{2 \gamma k_{z}^{2} \Delta_{ \pm}}{\Omega_{ \pm}}, \\
& \varepsilon_{1}^{( \pm)}\left(k_{x}, k_{y}\right)=\gamma_{1}\left(\frac{e E_{0}}{\hbar \omega}\right)^{2}+\gamma_{1} k^{2}+\xi_{ \pm}\left[\left(\hbar \omega-\Omega_{ \pm}\right.\right. \\
& \left.\left.-\frac{\gamma \Delta_{ \pm}\left(k_{x}^{2}+k_{y}^{2}\right)}{\Omega_{ \pm}}\right)^{2}+\frac{3 \gamma^{2}\left(\Omega_{ \pm} \mp \Delta_{ \pm}\right)^{2}\left(k_{x}^{2}+k_{y}^{2}\right)^{2}}{4 \Omega_{ \pm}^{2}}\right]^{1 / 2}, \\
& \varepsilon_{2}^{( \pm)}\left(k_{x}, k_{y}\right)=\gamma_{1}\left(\frac{e E_{0}}{\hbar \omega}\right)^{2}+\gamma_{1} k^{2}-\xi_{ \pm}\left[\left(\hbar \omega-\Omega_{ \pm}\right.\right. \\
& \left.\left.-\frac{\gamma \Delta_{ \pm}\left(k_{x}^{2}+k_{y}^{2}\right)}{\Omega_{ \pm}}\right)^{2}+\frac{3 \gamma^{2}\left(\Omega_{ \pm} \mp \Delta_{ \pm}\right)^{2}\left(k_{x}^{2}+k_{y}^{2}\right)^{2}}{4 \Omega_{ \pm}^{2}}\right]^{1 / 2},
\end{aligned}
$$

and $\xi_{ \pm}=\left(\hbar \omega-\Omega_{ \pm}\right) /\left|\hbar \omega-\Omega_{ \pm}\right|$. It should be stressed that Eqs. (24)-(25) correctly describe the electron energy spectrum near the band edge $\left(\mathbf{k}^{2} \ll \hbar \omega /|\gamma|\right)$ for any field frequency $\omega$. In the absence of the irradiation $\left(E_{0}=0\right)$, the effective Hamiltonian (21) turns into the unperturbed Luttinger Hamiltonian (3) and the solution of the secular equation (23) exactly coincides with the unperturbed electron dispersion, $\varepsilon^{( \pm)}(\mathbf{k})=\left(\gamma_{1} \pm 2 \gamma\right) k^{2}$.

\section{RESULTS AND DISCUSSION}

As it was mentioned above, the Luttinger Hamiltonian (2) can describe both valence band of conventional semiconductors (if the two quantities, $\gamma_{1} \pm 2 \gamma$, are of the same sign) and the band structure of gapless semiconductors near the band edge (if they are of opposite signs) since the both cases correspond to the same electronic term $\Gamma_{8}$ in the Brillouin zone center ${ }^{51}$. For definiteness, let us restrict the consideration by the cases of valence band of such a conventional semiconductor as $\operatorname{GaAs}\left(\gamma_{1}=-6.96 \hbar^{2} / 2 m_{0}, \gamma_{2}=-2.06 \hbar^{2} / 2 m_{0}\right.$, $\left.\gamma_{3}=-2.93 \hbar^{2} / 2 m_{0}\right)^{54}$ and the gapless semiconductor $\operatorname{HgTe}\left(\gamma_{1}=15.6 \hbar^{2} / 2 m_{0}, \gamma_{2}=9.6 \hbar^{2} / 2 m_{0}, \gamma_{3}=\right.$

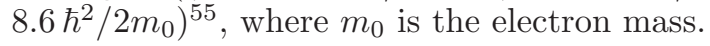

The energy spectrum of the term $\Gamma_{8}$ is defined by Eqs. (15) and (24)-(25) and plotted for GaAs (Fig. 2) and $\mathrm{HgTe}$ (Fig. 3) irradiated by an electromagnetic wave with different polarizations. In the absence of irradiation, the electronic term $\Gamma_{8}$ consists of the two branches which correspond to the bands of heavy and light holes in GaAs (see the red heavy lines in Fig. 2) and the conduction and valence bands in $\mathrm{HgTe}$ (see the red heavy lines in Fig. 3). These branches are degenerated at $\mathbf{k}=0$ and, in addition, their electron states are doubly degenerated
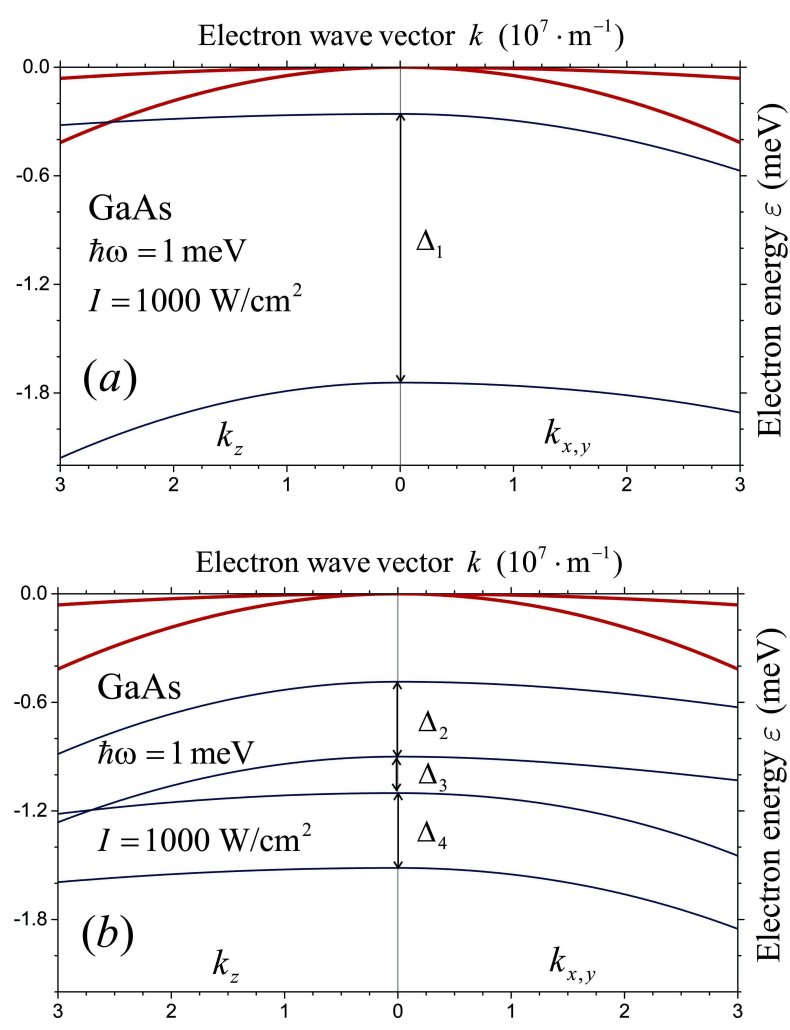

FIG. 2: Electron energy spectrum of GaAs sample, $\varepsilon(\mathbf{k})$, without irradiation (red heavy lines) and in the presence of an electromagnetic wave with the intensity $I=1000 \mathrm{~W} / \mathrm{cm}^{2}$, photon energy $\hbar \omega=1 \mathrm{meV}$ and different polarizations (blue thin lines): (a) linear polarization along the $z$ axis; (b) circular polarization in the $(x, y)$ plane.

in spin at any electron wave vector $\mathbf{k}$. It follows from the plots that the irradiation lifts the degeneracy but the lifting strongly depends on the light polarization. Namely, a linearly polarized wave splits the electron bands at $\mathbf{k}=0$ but does not lift the spin degeneracy of the bands (see the two blue thin lines in Figs. 1a and 2a), whereas a circularly polarized wave lifts also the spin degeneracy at any electron wave vector (see the four blue thin lines in Figs. 2b and 3b). It follows from Eqs. (15) and (24)(25) that the light-induced band splittings marked in the Figs. $2-3$ as $\Delta_{i}$ read

$$
\begin{aligned}
& \Delta_{1,5}=2|\gamma|\left(e E_{0} / \hbar \omega\right)^{2}, \\
& \Delta_{2,4,6,8}=\sqrt{\left[\gamma\left(e E_{0} / \hbar \omega\right)^{2}-\hbar \omega\right]^{2}+3 \gamma^{2}\left(e E_{0} / \hbar \omega\right)^{4}} \\
& +\sqrt{\left[\gamma\left(e E_{0} / \hbar \omega\right)^{2}+\hbar \omega\right]^{2}+3 \gamma^{2}\left(e E_{0} / \hbar \omega\right)^{4}}-2 \hbar \omega, \\
& \Delta_{3}=2 \hbar \omega-2 \sqrt{\left[\gamma\left(e E_{0} / \hbar \omega\right)^{2}+\hbar \omega\right]^{2}+3 \gamma^{2}\left(e E_{0} / \hbar \omega\right)^{4}}, \\
& \Delta_{7}=2 \hbar \omega-2 \sqrt{\left[\gamma\left(e E_{0} / \hbar \omega\right)^{2}-\hbar \omega\right]^{2}+3 \gamma^{2}\left(e E_{0} / \hbar \omega\right)^{4}} .
\end{aligned}
$$



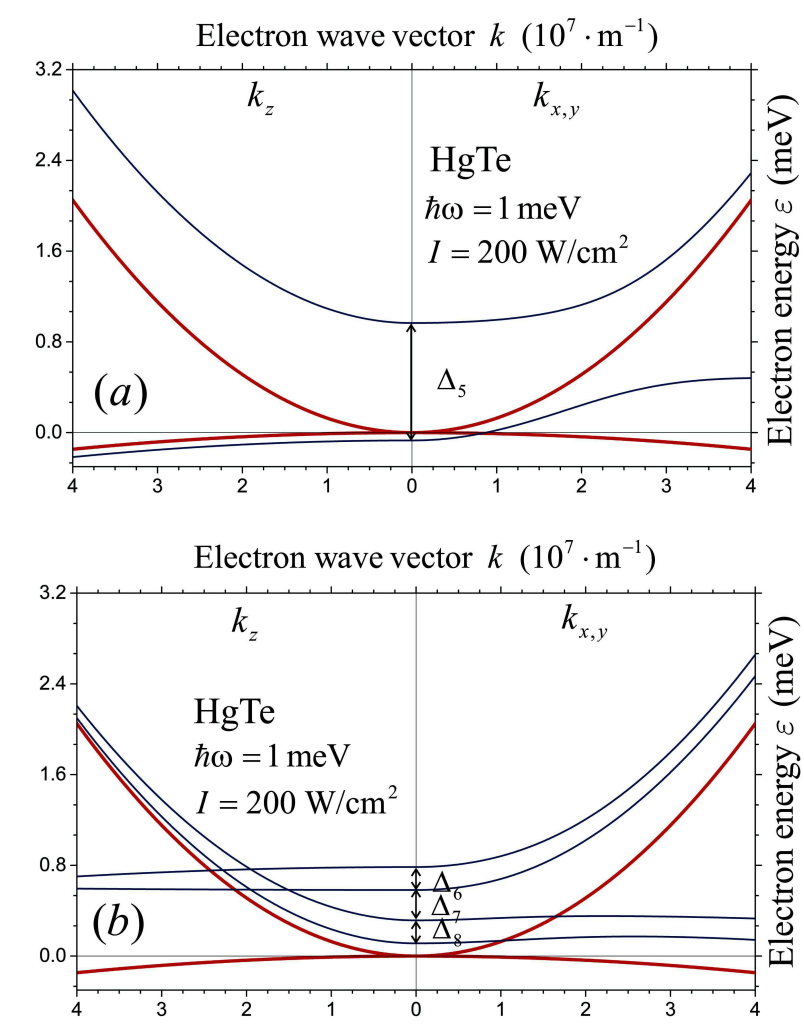

FIG. 3: Electron energy spectrum of HgTe sample, $\varepsilon(\mathbf{k})$, without irradiation (red heavy lines) and in the presence of an electromagnetic wave with the intensity $I=200 \mathrm{~W} / \mathrm{cm}^{2}$, photon energy $\hbar \omega=1 \mathrm{meV}$ and different polarizations (blue thin lines): (a) linear polarization along the $z$ axis; (b) circular polarization in the $(x, y)$ plane.

Since the band splittings (26) are of meV scale for the irradiation intensities around $I \sim \mathrm{kW} / \mathrm{cm}^{2}$, they can be observed experimentally in optical electron transitions induced by another weak (probing) electromagnetic wave. Particularly, such optical transitions between the split bands will lead to fine structure of the optical spectra. Besides the conventional optical measurements, the modern angle-resolved photoemission spectroscopy (ARPES) can also be applied to study the electron energy spectra plotted in Figs. 2-3. Indeed, ultraviolet laser-based ARPES provides sub-meV resolution (see, e.g., Refs. 56. 57), which is enough for detecting features of them. It should be noted also that the band splittings (26) appear from exact solutions of the Floquet problem at $\mathbf{k}=0$ and, therefore, go beyond the scope of the known simple mode ${ }^{58}$ based on the direct time-averaging of the Luttinger Hamiltonian.

To clarify physical nature of the light-induced band splitting, it should be noted that a circularly polarized electromagnetic wave breaks the time-reversal symmetry (since the time-reversal turns left-polarized photons into right-polarized ones and vice versa). Therefore, a circularly polarized electromagnetic wave acts similarly to a magnetic field which lifts the spin degeneracy and induced the asymmetry of electronic properties along the field direction and perpendicularly to the field. As to a linearly polarized electromagnetic wave, it acts similarly to an uniaxial mechanical stress along the direction of polarization vector, which both splits the degeneracy of electron states at $\mathbf{k}=0$ and induced the anisotropy of electron dispersion ${ }^{51}$. As a consequence, the light-induced band splitting is accompanied by the anisotropy of electronic properties. Indeed, the unperturbed electron dispersion, $\varepsilon^{( \pm)}(\mathbf{k})=\left(\gamma_{1} \pm 2 \gamma\right) k^{2}$, is isotropic, whereas an irradiation results in the anisotropy of electron dispersions (15) and (24)-(25) along different axes in the $\mathbf{k}$ space (see the blue thin lines in Figs. 2-3). Certainly, the anisotropy of electron dispersion will result in the anisotropy of electron transport which is discussed in the following.

Let charge carriers fill only ground band of the split bands near the band edge. Then the anisotropic transport can be described by effective electron masses. Expanding the electron energy spectra (15) and (24)-(25) into the series expansion in powers of electron wave vector $\mathbf{k}$, they can be easily rewritten near the band edge in the parabolic form,

$$
\varepsilon(\mathbf{k})=\frac{\hbar^{2}\left(k_{x}^{2}+k_{y}^{2}\right)}{2 m_{\perp}}+\frac{\hbar^{2} k_{z}^{2}}{2 m_{\|}}
$$

where $m_{\perp}$ and $m_{\|}$are the electron effective masses of the band. It should be stressed that the anisotropic electron dispersion (27) takes also place in valence band of conventional semiconductors under uniaxial mechanical stress. Therefore, we can apply the approach known from the theory of strained semiconductors, which is based on the the relaxation time approximation 51 . Within this approach, the conductivity tensor is

$$
\sigma_{\alpha \beta}=e^{2} \nu \int \frac{d^{3} \mathbf{k}}{(2 \pi)^{3}} v_{\alpha}(\mathbf{k}) v_{\beta}(\mathbf{k}) \tau(\varepsilon)\left[-\frac{\partial f_{0}(\varepsilon)}{\partial \varepsilon}\right],
$$

where $\varepsilon(\mathbf{k})$ is the electron energy spectrum (27) in the ground band, $\mathbf{v}(\mathbf{k})=\partial \varepsilon(\mathbf{k}) / \hbar \partial \mathbf{k}$ is the electron velocity, $\tau(\varepsilon)$ is the electron relaxation time, $f_{0}(\varepsilon)$ is the FermiDirac distribution function, and $\nu$ is the factor of spin degeneracy of the band ( $\nu=1,2$ for the cases of linear and circular polarizations, respectively). Assuming that the temperature is $T=0$, Eq. (28) yields the sought light-induced anisotropy of conductivity,

$$
\frac{\sigma_{z z}}{\sigma_{x x}}=\frac{\sigma_{z z}}{\sigma_{y y}}=\frac{m_{\perp}}{m_{\|}} .
$$

Particularly, for GaAs irradiated by a linearly polarized electromagnetic wave and the Fermi electron wave vector satisfying the condition $k_{F} \ll e E_{0} / \hbar \omega$, the anisotropy 
(29) does not depend on the field parameters and reads

$$
\frac{\sigma_{z z}}{\sigma_{x x}}=\frac{\sigma_{z z}}{\sigma_{y y}}=\frac{\gamma_{1}-2 \gamma}{\gamma_{1}+\gamma} .
$$

The theoretical studies of periodically driven systems described by the Luttinger Hamiltonian, which were based on approximate solutions of the Floquet problem, should also be noted ${ }^{59-61}$. Particularly, light-induced modifications of the electron bands originated from the Luttinger Hamiltonian were analyzed there as a series expansion in powers of $1 / \omega$. As a consequence, these results can be applied to real condensed-matter structures only in the high-frequency limit. In contrast to them, we found exact solutions of the Floquet problem with the Luttinger Hamiltonian at the band edge. Therefore, the present theory accurately describes all features of the electron dispersion near the band edge - the lightinduced band splitting, the light-induced anisotropy of effective masses, the effects depending on the light polarization, etc - in the broad frequency range, including low frequencies as well. To clarify the great importance of the low-frequency range for condensed-matter structures, it should be noted that the light-induced shift of electron energies in semiconductor materials is $\Delta \sim\left(e E_{0}\right)^{2} / m^{*} \omega^{2}$, where $m^{*}$ is the effective mass of charge carriers. To induce the experimentally observable shift $\Delta \sim \operatorname{meV}$ for infrared frequencies $(\hbar \omega \sim \mathrm{eV})$, an experimental sample should be irradiated by an electromagnetic wave with the giant intensity $I \sim \mathrm{GW} / \mathrm{cm}^{2}$, which can destruct the sample. However, the same energy shift $\Delta \sim$ meV can be achieved with a microwave electromagnetic field $(\hbar \omega \sim$ meV) with the reasonable intensity $I \sim \mathrm{kW} / \mathrm{cm}^{2}$. Therefore, the low-frequency irradiation is preferable from experimental viewpoint to observe the light-induced electronic features. Unfortunately, the microwave frequency range goes beyond the high-frequency approximation of the Floquet problem studied before and needs a special consideration. As a consequence, the low-frequency Floquet engineering of various condensed-matter structures became the exciting research area in the state-of-the-art Floquet theory (see, e.g., Ref. 62). Therefore, efforts to find solutions of the Floquet problem with the Luttinger Hamiltonian in the broad frequency range fit well the current tendencies in the condensed-matter physics.

It should be noted also that an analysis of the electronic term $\Gamma_{8}$ based on the tight-binding Hamiltonian is also possible. However, there is the native problem of all tight-binding Hamiltonians: The correct choice of basic (atomic) wave functions. Since interatomic matrix elements of the tight-binding Hamiltonian strongly depend on chosen atomic basis, it is not an easy task to compare the theoretical calculations with experimental data. On the contrary, the Luttinger Hamiltonian depends only on the three parameters $\gamma_{1,2,3}$ which are accurately found from many optical and transport measurements. Therefore, we believe that the approach based on the Luttinger Hamiltonian is preferable to study electronic states arisen from the $\Gamma_{8}$ term.

Finalizing the discussion, it should be reminded that the present theory is developed under assumption of continuous electron wave vector, $\mathbf{k}$. However, the effective Hamiltonians $\hat{\mathcal{H}}_{\text {eff }}(\mathbf{k})$ derived above can also be used to describe electronic properties of nanostructures, where the electron wave vector is discontinuous. To take into account the size quantization in nanostructures, one have to analyze the Schrödinger problem with the Hamiltonian $\hat{\mathcal{H}}_{\text {eff }}(\hat{\mathbf{k}})+U(\mathbf{r})$, where $\hat{\mathbf{k}}=-i \partial / \partial \mathbf{r}$ is the electron wave vector operator, $U(\mathbf{r})$ is the quantizing potential of the nanostructure, and the Hamiltonian $\hat{\mathcal{H}}_{\text {eff }}(\hat{\mathbf{k}})$ results from the effective Hamiltonians (14) and (21) with the replacement $\mathbf{k} \rightarrow \hat{\mathbf{k}}$.

\section{CONCLUSION}

Applying the Floquet formalism to electron states described by the Luttinger Hamiltonian, we developed the theory of optical control of the states originated from the electronic term $\Gamma_{8}$ (valence band in conventional semiconductors like GaAs and the valence and conduction bands in gapless semiconductors like HgTe). As a main result, exact solutions of the Floquet problem at the band edge are found and the electron energy spectrum of such materials renormalized by light is derived near the band edge for any field frequency. It follows from analysis of the spectrum that the electronic properties crucially depend on the irradiation which can induce the anisotropy of electronic properties for different directions in the Brillouin zone, band gaps in the spectrum and the spin splitting of the bands. Possible manifestations of the found electronic features in optical spectra and transport measurements are discussed. Since semiconductor materials described by the Luttinger Hamiltonian are actively used in the modern nanotechnology, the present theory can be helpful to describe electronic properties of various microand nanostructures.

\section{Acknowledgments}

The reported study was funded by RFBR (project number 20-02-00084). I.A.S. and O.V.K. thank Icelandic Science Foundation (project "Hybrid polaritonics"). I.A.S. thanks the Ministry of Science and Higher Education of the Russian Federation (Megagrant number 14.Y26.31.0015). 
tum Systems: Effective Hamiltonians and Engineered Gauge Fields, Phys. Rev. X 4, 031027 (2014).

2 M. Bukov, L. D'Alessio, and A. Polkovnikov, Universal high-frequency behavior of periodically driven systems: From dynamical stabilization to Floquet engineering, Adv. Phys. 64, 139 (2015).

${ }^{3}$ F. Casas, J. A. Oteo, and J. Ros, Floquet theory: exponential perturbative treatment, J. Phys. A 34, 3379 (2001).

4 A. Eckardt and E. Anisimovas, High-frequency approximation for periodically driven quantum systems from a Floquet-space perspective, New J. Phys. 17, 093039 (2015).

5 S. Rahav, I. Gilary, and S. Fishman, Effective Hamiltonians for Periodically Driven Systems, Phys. Rev. A 68, 013820 (2003).

6 M. Holthaus, Floquet engineering with quasienergy bands of periodically driven optical lattices, J. Phys. B 49, 013001 (2015).

7 C. Cohen-Tannoudji, J. Dupont-Roc, and G. Grynberg, Atom-Photon Interactions: Basic Processes and Applications (Wiley, Chichester, 1998).

8 S. H. Autler, . H. Townes, Stark Effect in Rapidly Varying Fields, Phys. Rev. 100, 703 (1955).

${ }^{9}$ V. M. Galitskii, S. P. Goreslavskii, V. F. Elesin, Electric and magnetic properties of a semiconductor in the field of a strong electromagnetic wave, JETP 30, 117 (1970).

10 S. P. Goreslavskii, V. F. Elesin, Electric properties of a semiconductor in the field of a strong electromagnetic wave, JETP Lett. 10, 316 (1969).

11 Q.T. Vu, H. Haug, O.D. Mücke, T. Tritschler, M. Wegener, G. Khitrova, H.M. Gibbs, Light-Induced Gaps in Semiconductor Band-to-Band Transitions, Phys. Rev. Lett. 92, 217403 (2004).

12 J. Z. Kaminski, On the laser-induced modification of the energy band structure, Acta Physica Polonica A 83, 495 (1993).

13 Y. Mizumoto, Y. Kayanuma, Band renormalization of semiconductors by high-intensity infrared laser: Onedimensional model, Phys. Rev. B 72, 115203 (2003).

14 A. Srivastava, R. Srivastava, J. Wang, J. Kono, LaserInduced Above-Band-Gap Transparency in GaAs, Phys. Rev. Lett. 93, 157401 (2004).

15 Y. Mizumoto, Y. Kayanuma, Dressed-band theory for semiconductors in a high-intensity infrared laser field, Phys. Rev. B 74, 045216 (2006).

16 H. Lignier, C. Sias, D. Ciampini, Y. Singh, A. Zenesini, O. Morsch, E. Arimondo, Dynamical Control of MatterWave Tunneling in Periodic Potentials, Phys. Rev. Lett. 99, 220403 (2007).

17 Y. Mizumoto, Y. Kayanuma, Dynamical collapse of the Peierls gap by an intense laser field, Phys. Rev. B 81, 233202 (2010).

18 S. Ghimire, A. D. DiChiara, E. Sistrunk, U. B. Szafruga, P. Agostini, L. F. DiMauro and D. A. Reis, Redshift in the Optical Absorption of $\mathrm{ZnO}$ Single Crystals in the Presence of an Intense Midinfrared Laser Field, Phys. Rev. Lett. 107, 167407 (2010)

19 O. V. Kibis, Dissipationless electron transport in photondressed nanostructures, Phys. Rev. Lett. 107, 106802 (2011).

20 O. V. Kibis, O. Kyriienko, I. A. Shelykh, Persistent current induced by vacuum fluctuations in a quantum ring, Phys. Rev. B 87, 245437 (2013).

${ }^{21}$ H. Sigurdsson, O. V. Kibis, I. A. Shelykh, Optically in- duced Aharonov-Bohm effect in mesoscopic rings, Phys. Rev. B 90, 235413 (2014).

22 O. V. Kibis, H. Sigurdsson, I. A. Shelykh, Aharonov-Bohm effect for excitons in a semiconductor quantum ring dressed by circularly polarized light, Phys. Rev. B 91, 235308 (2015).

23 M. Hasan, I. V. Iorsh, O. V. Kibis, I. A. Shelykh, Optically controlled periodical chain of quantum rings, Phys. Rev. B 93, 125401 (2016)

24 V. K. Kozin, I. V. Iorsh, O. V. Kibis, I. A. Shelykh, Quantum ring with the Rashba spin-orbit interaction in the regime of strong light-matter coupling, Phys. Rev. B 97, 155434 (2018).

25 V. K. Kozin, I. V. Iorsh, O. V. Kibis, I. A. Shelykh, Periodic array of quantum rings strongly coupled to circularly polarized light as a topological insulator, Phys. Rev. B 97, 035416 (2018).

26 O. V. Kibis, Persistent current induced by quantum light, Phys. Rev. B 86, 155108 (2012).

27 O. V. Kibis, How to suppress the backscattering of conduction electrons?, Europhys. Lett. 107, 57003 (2014).

28 S. Morina, O. V. Kibis, A. A. Pervishko, I. A. Shelykh, Transport properties of a two-dimensional electron gas dressed by light, Phys. Rev. B 91, 155312 (2015).

29 A. A. Pervishko, O. V. Kibis, S. Morina, I. A. Shelykh, Control of spin dynamics in a two-dimensional electron gas by electromagnetic dressing, Phys. Rev. B 92, 205403 (2015).

30 K. Dini, O. V. Kibis, I. A. Shelykh, Magnetic properties of a two-dimensional electron gas strongly coupled to light, Phys. Rev. B 93, 235411 (2016).

${ }^{31}$ H. K. Avetissian, G. F. Mkrtchian, Nonlinear response of the quantum Hall system to a strong electromagnetic radiation, Phys. Lett. A 380, 3924 (2016).

32 O. Kyriienko, O. V. Kibis, I. A. Shelykh, Floquet control of dipolaritons in quantum wells, Opt. Lett. 42, 2398 (2017).

33 Y. H. Wang, H. Steinberg, P. Jarillo-Herrero, and N. Gedik, Observation of Floquet-Bloch states on the surface of a topological insulator, Science 342, 453 (2013).

${ }^{34}$ L. E. F. Foa Torres, P. M. Perez-Piskunow, C. A. Balseiro, G. Usaj, Multiterminal Conductance of a Floquet Topological Insulator, Phys. Rev. Lett. 113, 266801 (2014).

35 G. Usaj, P. M. Perez-Piskunow, L. E. F. Foa Torres, C. A. Balseiro, Irradiated graphene as a tunable Floquet topological insulator, Phys. Rev. B 90, 115423 (2014).

${ }^{36}$ H. L. Calvo, L. E. F. Foa Torres, P. M. Perez-Piskunow, C. A. Balseiro, G. Usaj, Floquet interface states in illuminated threedimensional topological insulators, Phys. Rev. B 91, 241404(R) (2015).

37 T. Mikami, S. Kitamura, K. Yasuda, N. Tsuji, T. Oka, H. Aoki, Brillouin-Wigner theory for high-frequency expansion in periodically driven systems: Application to Floquet topological insulators, Phys. Rev. B 93, 144307 (2016).

${ }^{38}$ D. Yudin, O. V. Kibis, I. A. Shelykh, Optically tunable spin transport on the surface of a topological insulator, New J. Phys. 18, 103014 (2016).

39 O. Kyriienko, O. V. Kibis, I. A. Shelykh, Optically induced topological states on the surface of mercury telluride, Phys. Rev. B 99, 115411 (2019).

40 T. Oka, H. Aoki, Photovoltaic Hall effect in graphene, Phys. Rev. B 79, 081406 (2009).

41 O.V. Kibis, Metal-insulator transition in graphene induced by circularly polarized photons, Phys. Rev. B 81, 165433 (2010). 
42 S. V. Syzranov, Ya. I. Rodionov, K. I. Kugel, F. Nori, Strongly anisotropic Dirac quasiparticles in irradiated graphene, Phys. Rev. B 88, 241112 (2013).

43 P. M. Perez-Piskunow, G. Usaj, C. A. Balseiro, L. E. F. Foa Torres, Floquet chiral edge states in graphene, Phys. Rev. B 89, 121401(R) (2014).

44 K. Kristinsson, O. V. Kibis, S. Morina, I. A. Shelykh, Control of electronic transport in graphene by electromagnetic dressing, Sci. Rep. 6, 20082 (2016).

45 O. V. Kibis, S. Morina, K. Dini, I. A. Shelykh, Magnetoelectronic properties of graphene dressed by a highfrequency field, Phys. Rev. B 93, 115420 (2016).

46 O. V. Kibis, K. Dini, I. V. Iorsh, I. A. Shelykh, All-optical band engineering of gapped Dirac materials, Phys. Rev. B 95, 125401 (2017).

47 I. V. Iorsh, K. Dini, O. V. Kibis, I. A. Shelykh, Optically induced Lifshitz transition in bilayer graphene, Phys. Rev. B 96, 155432 (2017).

48 A. Iurov, G. Gumbs, D. H. Huang, Peculiar electronic states, symmetries, and Berry phases in irradiated alphaT(3)materials, Phys. Rev. B 99, 205135 (2019).

49 A. Iurov, L. Zhemchuzhna, D. Dahal, G. Gumbs, and D. Huang, Quantum-statistical theory for laser-tuned transport and optical conductivities of dressed electrons in alpha-T(3)materials, Phys. Rev. B 101, 035129 (2020).

50 J. M. Luttinger, Quantum Theory of Cyclotron Resonance in Semiconductors: General Theory, Phys. Rev. 102, 1030 (1956).

51 G. L. Bir and G. E. Pikus, Symmetry and Strain-Induced Effects in Semiconductors (Wiley, New York, 1974).

${ }^{52}$ M. I. D'yakonov and A. V. Khaetskii, Surface states in a gapless semiconductor, JETP Lett. 33, 110 (1981).
${ }^{53}$ O. V. Kibis, O. Kyriienko, I. A. Shelykh, New J. Phys. 21, 043016 (2019).

54 S. Adachi, GaAs and Related Materials: Bulk Semiconducting and Superlattice Properties (World Scientific, Singapore, 1994).

55 S. Adachi, Handbook of Physical Properties of Semiconductors: Vol. 3, II-VI Compound Semiconductors (Kluwer, New York, 2004).

56 W. Zhang, Photoemission Spectroscopy on High Temperature Superconductor (Springer, Berlin, 2013).

57 T. Kiss, F. Kanetaka, T.Yokoya, T. Shimojima, K. Kanai, S. Shin, Y. Onuki, T. Togashi, C. Zhang, C. T. Chen, and S. Watanabe, Photoemission Spectroscopic Evidence of Gap Anisotropy in an f-Electron Superconductor, Phys. Rev. Lett. 94, 057001 (2005).

58 Y. T. Rebane, Semiconductor and dielectric energy-band reconstruction in a field of intensive weal self-absorbing light waves, Fiz. Tverd. Tela 27, 1364 (1984).

59 D. Zhang, H. Wang, J. Ruan, G. Yao, and H. Zhang, Engineering topological phases in the Luttinger semimetal $\alpha$ Sn, Phys. Rev. B 97, 195139 (2018)

60 S. A. A. Ghorashi, P. Hosur, and C.-S. Ting, Irradiated three-dimensional Luttinger semimetal: A factory for engineeringWeyl semimetals, Phys. Rev. B 97, 205402 (2018).

61 S. A. A. Ghorashi, Hybrid Dispersion Dirac Semimetal and Hybrid Weyl Phases in Luttinger Semimetals: A Dynamical Approach, Annalen der Physik, 5321900336 (2020).

62 M. Vogl, M. Rodriguez-Vega, and G. A. Fiete, Effective Floquet Hamiltonian in the low-frequency regime, Phys. Rev. B 101, 024303 (2020) 